\title{
OS BENEFÍCIOS DA IMPLANTAÇÃO DA GASTRONOMIA HOSPITALAR PARA OPÚBLICO ADULTO: UMA REVISÃO BIBLIOGRÁFICA
}

\author{
Espedita Lopes ${ }^{1}$ \\ Maudelan Santos ${ }^{2}$ \\ Nathan Barbosa ${ }^{3}$ \\ Ângelo Almeida ${ }^{4}$ \\ Diogenes José Gusmão Coutinho 5
}

RESUMO: O agravamento do estado nutricional durante a internação hospitalar, afeta negativamente o estado clínico do paciente, ocorrendo alterações fisiológicas no processo de adaptação do organismo com a falta de nutrientes. Com isso o cuidado centrado no paciente ajuda a recuperar rapidamente e a manter a saúde e o bem-estar. Evitar a desnutrição de pacientes hospitalizados sempre foi uma das preocupações dos departamentos de nutrição e alimentação, buscando oferecer cardápios que atendam às necessidades fisiológicas e sensoriais das refeições, analisando os benefícios da nutrição e alimentação. Este estudo trata-se de uma revisão abrangente da literatura com o intuito de encontrar através de pesquisas realizadas em bases de dados o conhecimento existente sobre o assunto. Atualmente, a atuação da Nutrição na elaboração de cardápios saudáveis e na manutenção e recuperação do estado nutricional dos pacientes hospitalizados tem encontrado aliados na indústria gastronômica. Conclui-se que a introdução da tecnologia gastronômica é propícia a uma maior aceitação das refeições fornecidas para os pacientes, devido a qualidade da apresentação e do sabor, aumentando a ingestão, propício à melhoria do estado nutricional dos pacientes hospitalizados, reduzindo a desnutrição e otimizando os serviços para diminuir custos edesperdícios, relacionados para a reabilitação de pacientes internados. por isso a nutrição é essencial para a sobrevivência de qualquer espécie, responsável por garantir os nutrientes necessários. Como ciência, ela estuda a composição dos alimentos e avalia as necessidades nutricionais dos indivíduos, seja para manter a saúde para tratar alguma doença. O nutricionista precisa conhecer a necessidade do paciente, inseri-lo em um mundo novo para que ele possa aprender a se alimentar, ensinar sobre os alimentos e de que forma inseri-los no dia a dia do paciente.

Palavras-chave : Desnutrição. Dietoterapia. Gastronomia. Nutrição. serviços de alimentação.

ABSTRACT: The worsening of the nutritional status during hospitalization negatively affects the patient's clinical status, with physiological alterations occurring in the body's

\footnotetext{
${ }^{\mathrm{I}}$ Graduanda em nutrição do Centro Universitário Tiradentes, Recife/PE.

${ }^{2}$ Graduanda em nutrição do Centro Universitário Tiradentes, Recife/PE.

${ }^{3}$ Graduando em nutrição do Centro Universitário Tiradentes, Recife/PE.

${ }^{4}$ Professor orientador do curso de nutrição do Centro UniversitárioTiradentes, Recife/PE.

${ }^{5}$ Professor Doutor em Biologia pela UFPE.
} 
process of adaptation to the lack of nutrients. Thus, patient-centered care helps to quickly recover and maintain health and well-being. Avoiding malnutrition in hospitalized patients has always been one of the concerns of nutrition and feeding departments, seeking to offer menus that meet the physiological and sensory needs of meals, analyzing the benefits of nutrition and feeding. This work is a comprehensive review of the literature in order to find through research conducted in databases the existing knowledge on the subject. Currently, the performance of Nutrition in the development of healthy menus and the maintenance and recovery of the nutritional status of hospitalized patients has found allies in the gastronomy industry. It is concluded that the introduction of gastronomic technology is conducive to a greater acceptance of the meals provided to patients, due to the quality of presentation and taste, increasing the intake, conducive to improving the nutritional status of hospitalized patients, reducing malnutrition and optimizing services to reduce costs and waste, related to the rehabilitation of inpatients. so nutrition is essential for the survival of any species, responsible for ensuring the necessary nutrients. As a science, it studies the composition of food and assesses the nutritional needs of individuals, either to maintain health or to treat a disease. The nutritionist needs to know the patient's needs, to insert him into a new world so that he can learn to eat, to teach about food, and how to insert it into the patient's daily routine.

Keywords: Diet therapy. food services. Gastronomy. malnutrition. nutrition.

\section{INTRODUÇÃO}

A gastronomia é uma parte da culinária que abrange conhecimentos e práticas que

estão ligadas com a alimentação. Já a gastronomia hospitalar apareceu para aumentar a aceitação das refeições oferecidas aos pacientes, usando técnicas científicas para construir pratos nutricionalmente equilibrados e auxiliando na recuperação da patologia que o paciente apresenta, ela trabalha para que todos os aspectos visuais, olfativos e gustativos sejam estimulados para que os indivíduos que consomem esses pratos sintam prazerosos (SOUZA; MOLERO; MOLINA, 202I).

Segundo Lima (2018) ela permite técnicas criativas para apresentação das refeições, bem como combinações de diferentes temperos que modifiquem o sabor da preparação e preserve a cultura alimentar e ambiente social em que o indivíduo hospitalizado está inserido, de modo a considerar que os pacientes não estão em seu convívio social e familiar, que, por sua vez, pode influenciar negativamente na ingestão alimentar.

A Nutrição e a Gastronomia elas se complementam e conjuntamente trazem benefícios e qualidade de vida ao indivíduo, sendo a junção de hábitos saudáveis e prazerosos no ato de se alimentar (MARTINS; BARATTO, 2018). Mudando os conceitos e as atitudes sobre as dietas servidas, trabalhando com todos os aspectos sensoriais dos alimentos como a cor, o aroma, o sabor, a textura e a temperatura (SANTOS; GONTIJO; 
ALMEIDA, 2017).

É pensando na nutrição adequada como meio importante para a recuperação do paciente, agregando valores ao atendimento humanizado e multidisciplinar ao paciente hospitalizado. Com oferta atrativa de refeições equilibradas e que sejam agradáveis ao paladar e ao apelo visual possibilitam maior aceitação da dieta favorecendo uma experiência de satisfação no momento de se alimentar(DELGADO, 2017).

No hospital a concepção da alimentação e do alimento pelos profissionais da saúde e da nutrição ainda é considerada restrita ao ponto de vista técnico-científico e às dimensões higiênico-sanitária e nutricional, o que faz a alimentação hospitalar ser popularmente vista como monótona, restritiva e sem sabor, sendo frequentemente rejeitada pelos pacientes (SPENCE, 2017).

Segundo Paiva (2018) a Nutrição é o estudo da ciência que investiga as relações entre o alimento ingerido pelo indivíduo $e$ as doenças, buscando o bem-estar $e$ a preservação da saúde humana. Desse modo, pode- se dizer que a nutrição constitui um conjunto de fenômenos físicos, químicos, físico e fisiológicos que se passam no interior do organismo e mediante os quais este recebe e utiliza os materiais fornecidos pelos alimentos - isto é, os nutrientes, os quais são necessários para a formação e manutenção de toda matéria viva no nosso organismo.

A gastronomia hospitalar concilia a prescrição dietética e com elaboração de refeições atrativas e saborosas, a fim de promover a nutrição com prazer e ainda a apresentação do prato e da bandeja, a variedade do cardápio, o treinamento especializado dos colaboradores, a cordialidade no atendimento e a rapidez do serviço (PINTO, ALVES 2017).

A partir destas iniciativas propiciar um ambiente demonstrando empatia, gerando confiança, respeito, que são tão importantes quanto os tratamentos ao apoio humano nesta fase. Pensando na nutrição adequada como meio importante para a recuperação, agregando valor ao atendimento humanizado e multidisciplinar ao paciente hospitalizado. Como oferta atrativa de refeições equilibradas e que sejam agradáveis ao paladar, e ao apelo visual possibilitando maior aceitação da dieta favorecendo uma experiência de satisfação no momento de se alimentar (DELGADO, 2017).

O objetivo deste trabalho é realizar uma revisão da literatura bibliográfica sobre a culinária hospitalar que surgiu como ferramenta para o bem-estar do paciente em 1994 . 
Hábitos alimentares regionais observados durante o estágio clínico de nutrição na Universidade Tiradentes (UNIT) para determinar a possibilidade de ação humanitária. No campo da alimentação e gastronomia, fortalecer e promover um ambiente mais positivo propício a uma melhor recuperaçãodos pacientes internados.

\section{METODOLOGIA}

O Presente estudo é caracterizado como estudo bibliográfico, de caráter descritivo com análise de referência obtidas nas seguintes bases de dados: as bases essenciais Literature Analysis And Retrieval System online/PubMed (Medline) e literatura Científica e Técnica da América Latina e Caribe/BVS-Biblioteca Virtual em Saúde (LILACS), a base opcional Scientific Electronic Library Online (Scielo), buscadores na web e literatura cinzenta (busca manual). Para identificar todas as publicações relevantes, foram realizadas is buscas de artigos científicos iniciadas em julho de 202I e finalizadas em outubro de 2021, com base nos artigos disponíveis nos últimos cinco anos.

A estratégia de busca foi definida por descritores cadastrados nos Descritores em Ciências da Saúde (DECs), com o unitermo "protocolo" em combinação com termos relativos a modificação da dieta, desnutrição, gastronomia hospitalar e aceitação dos pacientes, para busca da pesquisa foram utilizadas as palavras chave (Desnutrição, dietoterapia, gastronomia, nutrição, serviços de alimentação). Os critérios de inclusão foram definidos previamente: artigos redigidos nos idiomas portugûes, e inglês, benefícios da gastronomia hospitalar, estudos realizados em paciente hospitalar; em manuscrito publicados de 2017 a 2021. Os critérios de exclusão de artigos compreendem: artigos de revisão não disponíveis na versão completa, comunicações breves e artigos, os benefícios da implantação da gastronomia hospitalar; artigos indisponíveis quando solicitados aos autores; que não referisse aos critérios definidos para a inclusão. A seleção dos estudos foi realizada identificando o total de referências da busca de dados nas bases eletrônicas e na base manual, excluindo as duplicadas e em sequência, uma triagempor meio da leitura do título e resumo.

As referências selecionadas foram analisadas por completo, nas quais, foi feita a extração dos dados (autor, ano de publicação, amostra de estudos, objetivo). Os manuscritos foram avaliados quanto à principal questão da pesquisa: Os benefícios da implantação da gastronomia hospitalar em público adulto. Todos os artigos que se 
enquadram com o desenho do estudo, foram incluídos na avaliação e selecionados a fim de estabelecer dados e materiais de análise relevantes para a construção do artigo

\subsection{RESULTADOS E DISCUSSÃO}

Quadro I - caracterização dos artigos

\begin{tabular}{|c|c|c|}
\hline AUTOR & TÍTULO & RESULTADO \\
\hline $\begin{array}{l}\text { Behrmann } \\
\text { e } \\
\text { a Lim } \\
\text { (2019) }\end{array}$ & 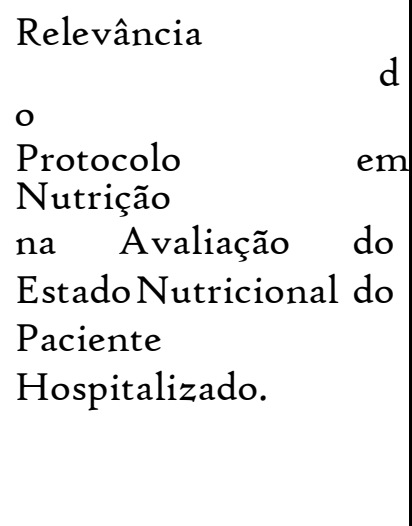 & $\begin{array}{l}\text { Aborda a dieta hospitalar afirmando que os } \\
\text { pacientes hospitalizados frequentemente } \\
\text { apresentam condições potenciais para a } \\
\text { desnutrição que podem ocorrer de forma } \\
\text { mais agressiva e acelerada por possuírem } \\
\text { como agravante a própria condição } \\
\text { clínica. }\end{array}$ \\
\hline $\begin{array}{l}\text { Gonçalves } \\
\text { et al. } \\
(2018)\end{array}$ & $\begin{array}{l}\text { A importância } \\
\text { da } \\
\text { Gastronomia para a } \\
\text { nutrição. }\end{array}$ & $\begin{array}{l}\text { Descreve os benefícios de combinar nutrição } \\
\text { e culinária e mostra aos pacientes e } \\
\text { consumidores que podem se alimentar de } \\
\text { forma saudável sem abrir mão da praticidade } \\
\text { e do sabor. A combinação de nutrição e } \\
\text { alimentação é uma realidade iminente, pois } \\
\text { saúde e alimentação são um binômio } \\
\text { intimamente relacionado. }\end{array}$ \\
\hline $\begin{array}{l}\text { Martins } \\
\text { Baratto } \\
(2018)\end{array}$ & $\begin{array}{l}\text { Gastronomia } \\
\text { hospitalar: } \\
\text { treinamento } \\
\text { bases de cozinha }\end{array}$ & $\begin{array}{l}\text { As técnicas de hotelaria e gastronomia estão } \\
\text { sendo estudadas por gestores hospitalares e } \\
\text { então inseridas ao serviço. O objetivo deste } \\
\text { estudo foi repassar conhecimento } \\
\text { gastronômico, com pequenas adaptações ao } \\
\text { âmbito hospitalar, mantendo as propriedades } \\
\text { dos alimentos, prezando por sua aparência } \\
\text { final como prato apetitoso, e assim fazer a } \\
\text { inclusão da gastronomia denominada } \\
\text { hospitalar. }\end{array}$ \\
\hline $\begin{array}{l}\text { Melo } \\
(2018)\end{array}$ & $\begin{array}{l}\text { Gastronomia } \\
\text { hospitalar e relações } \\
\text { de hospitalidade: } \\
\text { estudo de casos } \\
\text { múltiplo na prestação } \\
\text { de serviços } \\
\quad \text { de }\end{array}$ & $\begin{array}{l}\text { As relações de hospitalidade e a gastronomia } \\
\text { hospitalar configuram-se como uma } \\
\text { estratégia fundamental para manter a } \\
\text { satisfação e fidelização de clientes. Foi } \\
\text { comprovado que a gastronomia influencia } \\
\text { nas relações de hospitalidade entre o setor }\end{array}$ \\
\hline
\end{tabular}




\begin{tabular}{|c|c|c|}
\hline & $\begin{array}{l}\text { hospitais } \\
\text { ludovicenses }\end{array}$ & de nutrição e os clientes de saúde. \\
\hline $\begin{array}{l}\text { Pinto } \\
\text { Alves } \\
(2017)\end{array}$ & $\begin{array}{l}\text { A gastronomia } \\
\text { no } \\
\text { contexto da hotelaria } \\
\text { hospitalar: um estudo } \\
\text { de caso na cidade do } \\
\text { Rio de Janeiro, RJ, } \\
\text { Brasil }\end{array}$ & $\begin{array}{l}\text { As organizações de saúde buscam inserir a } \\
\text { gastronomia no âmbito hospitalar, no intuito } \\
\text { de garantir a satisfação e fidelizar os clientes, } \\
\text { aderindo e conciliando as ferramentas da } \\
\text { indústria de serviços, concebendo um novo } \\
\text { conceito de bem receber. A gastronomia está } \\
\text { associada à habilidade no preparo dos } \\
\text { alimentos, tornando as refeições mais } \\
\text { saborosas, estimulando o prazer pela } \\
\text { refeição. }\end{array}$ \\
\hline $\begin{array}{l}\text { Lobato } \\
\text { (2019) }\end{array}$ & $\begin{array}{l}\text { distribuição } \\
\text { e } \\
\text { refeições em âmbito } \\
\text { hospitalar. }\end{array}$ & $\begin{array}{l}\text { A Unidade de Alimentação e Nutrição } \\
\text { hospitalar deve estar apta a distribuir } \\
\text { refeições isentas de qualquer contaminante } \\
\text { (físico, químico ou biológico), além de } \\
\text { praticar com exatidão as condutas médicas e } \\
\text { dietéticas prescritas, envolvendo também, } \\
\text { técnicas de porcionamento e a coesa prática } \\
\text { entre dieta prescrita e distribuição de } \\
\text { refeições }\end{array}$ \\
\hline $\begin{array}{l}\text { Paiva } \\
\text { (2018) }\end{array}$ & $\begin{array}{l}\text { Gastronomia } \\
\text { e } \\
\text { nutrição: perspectiva } \\
\text { pessoal e profissional }\end{array}$ & $\begin{array}{l}\text { Gastronomia tem relação direta com a área } \\
\text { de alimentação coletiva, poderia ser melhor } \\
\text { explorado nas universidades em termos de } \\
\text { pesquisa. Ressaltando que diante desse } \\
\text { aprendizado sobre gastronomia muitas } \\
\text { possibilidades surgiram }\end{array}$ \\
\hline (2018) & 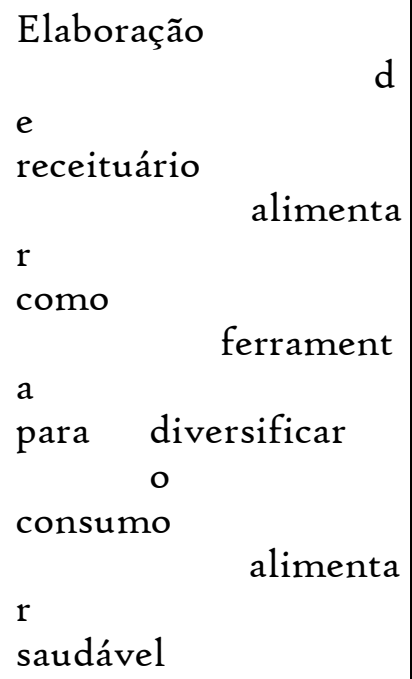 & $\begin{array}{l}\text { A nutrição e a gastronomia estão cada vez } \\
\text { mais ligadas, pois estão cada vez mais em } \\
\text { busca, de desenvolver um cardápio saboroso } \\
\text { e com alto valor nutritivo, além de, manter o } \\
\text { compromisso com a sustentabilidade } \\
\text { mediante o aproveitamento e }\end{array}$ \\
\hline
\end{tabular}


reaproveitamento de alimentos.

A manutenção e recuperação do estado nutricional de pacientes hospitalizados será o foco principal dos nutricionistas, e agora existem formas mais abrangentes de agregar à sua dietoterapia produtos saudáveis, nutritivos, saborosos e principalmente atrativos. A nutrição e a gastronomia hospitalar agregam valor histórico a essa situação e levam a melhorias contínuas na assistência nutricional, fazendo com que os pacientes sigam mais o plano alimentar sem ignorar o sabor da dieta (MARTINS; BARATTO, 2018)

\subsection{GASTRONOMIA HOSPITALAR}

Ofertados aos pacientes com a qualidade dos aspectos simbólicos e sensoriais (sabor, cheiro, temperatura, aparência) que deve ser levada em consideração em uma dieta hospitalar, pois os pacientes hospitalizados julgam-na importante, o que pode determinar o grau de satisfação e, consequentemente, maior adesão ao tratamento proposto (MENDES, 2019). Os profissionais de nutrição têm investido bastante na gastronomia hospitalar para conseguir mudar o velho conceito de que comida de hospital é horrível e sem graça (MARTINS; BARATTO, 20I8).A dieta hospitalar precisa estar adequada às condições de saúde de quem está internado. Levando tudo isso em conta, as equipes médicas e de nutrição clínica escolhem qual das opções de dieta é melhor para o paciente (TALDIVO, 20I6).

Segundo Taldivo (2016) as dietas hospitalares podem ser: Dieta geral, ela é indicada para pessoas que não tem complicações de saúde, Dieta branda são- Alimentos mais cozidos do que o normal, com teor mínimo de gordura e sem fritura. Indicada para problemas digestivos em geral e para o pós-operatório. Dieta pastosa são alimentos em forma de papa, geralmente ela é hipossódica, para pacientes com problemas cardiovasculares, hipertensos e com dificuldade de deglutição (disfagia). Dieta leve facilita a digestão e o esvaziamento do aparelho digestivo.

A Unidade de Alimentação e Nutrição hospitalar deve estar apta a distribuir refeições isentas de qualquer contaminante (físico, químico ou biológico), além de praticar com exatidão as condutas médicas e dietéticas prescritas, envolvendo também, técnicas de porcionamento e a coesa prática entre dieta prescrita e distribuição de refeições (LOBATO, 2019). 
Rigo et al., (2020) foi realizado um estudo com homens e mulheres internados em duas unidades hospitalares, com objetivo de avaliar a aceitabilidade das refeições do almoço de pacientes que receberam a dietética para Diabetes Mellitus (DM) e verificar aspectos que podem interferir nesse consumo, como as estações do ano. A pesquisa foi realizada em duas etapas, a primeira etapa foi no verão e a segunda etapa no inverno. Com relação às refeições, observou-se que na primeira etapa, os alimentos menos consumidos foram arroz branco, brócolis cozido, feijão preto e carne bovina; e na segunda etapa, os menos consumidos foram carne bovina, frango, feijão preto e arroz branco. As preparações com maior índice de rejeição foram feijão preto, brócolis cozido, carne bovina, arroz branco, beringela, cenoura e chuchu cozidos. O desperdício nas refeições do almoço apresentou média per capita de 64,15g no verão e de 135,99g no inverno. A aceitação de frutas e sucos foi maior no período do verão (82\%) quando comparado ao período do inverno $(63 \%)$. Os autores identificaram que esses foram os alimentos que mais se repetiam no cardápio, sugerindo que a falta de variedade das preparações pode causar desinteresse pela refeição.

\subsection{NUTRIÇÃO E GASTRONOMIA}

A gastronomia hospitalar é um método nutricional que visa melhorar a relaçãode paciente em tratamento com a dieta hospitalar. Com esta função, é possível ajustar a alimentação de acordo com as necessidades e preferências de cada paciente, e mudar a noção de comida ruim nos hospitais, as unidades alimentares muitas vezes dão prioridade ao tratamento, em casos mais simples, e acabam esquecendo-se de dá prioridade, aos casos mais graves na hora realização das refeições hospitalares.

A base da culinária é atender às necessidades nutricionais de cada patologia, buscando oferecer alimentos mais atrativos. É importante aplicar várias tecnologias juntas. De nada adianta ter muitos profissionais dessa área se você não tiver uma dieta que atraia mais os pacientes do setor hospitalar. Sem alimentos saudáveis e saborosos, eles não trarão resultados positivos aos pacientes.

O uso de alimentos na nutrição ainda é uma realidade pouco conhecida, mas está crescendo e se desenvolvendo. Muitos hospitais perceberam que essa demanda é um dos fatores que podem melhorar seus serviços e aumentar a fidelização de clientes. 
Apesar da evolução dos conceitos de hotelaria e gastronomia hospitalar observada nos últimos anos, sendo as dietas hospitalares alvo de mudanças no cenário atual, e da grande influência da alimentação na recuperação de pacientes hospitalizados.

É importante também que sejam feitos estudos que possam investigar o impacto da melhor aceitação provocada pela gastronomia no estado nutricional dos pacientes, já que, como evidenciado durante o desenvolvimento deste trabalho, a desnutrição é um problema de saúde pública no ambiente hospitalar que causa sérios prejuízos na reabilitação dos enfermos, sendo importante lançar mão do maior número de estratégias possível para reduzir seus índices.

Dessa forma, além de tornar o hospital um ambiente mais acolhedor e minimizar o impacto da internação sobre os pacientes, esses novos conceitos poderão ser aplicados de forma a contribuir para a redução da desnutrição hospitalar.

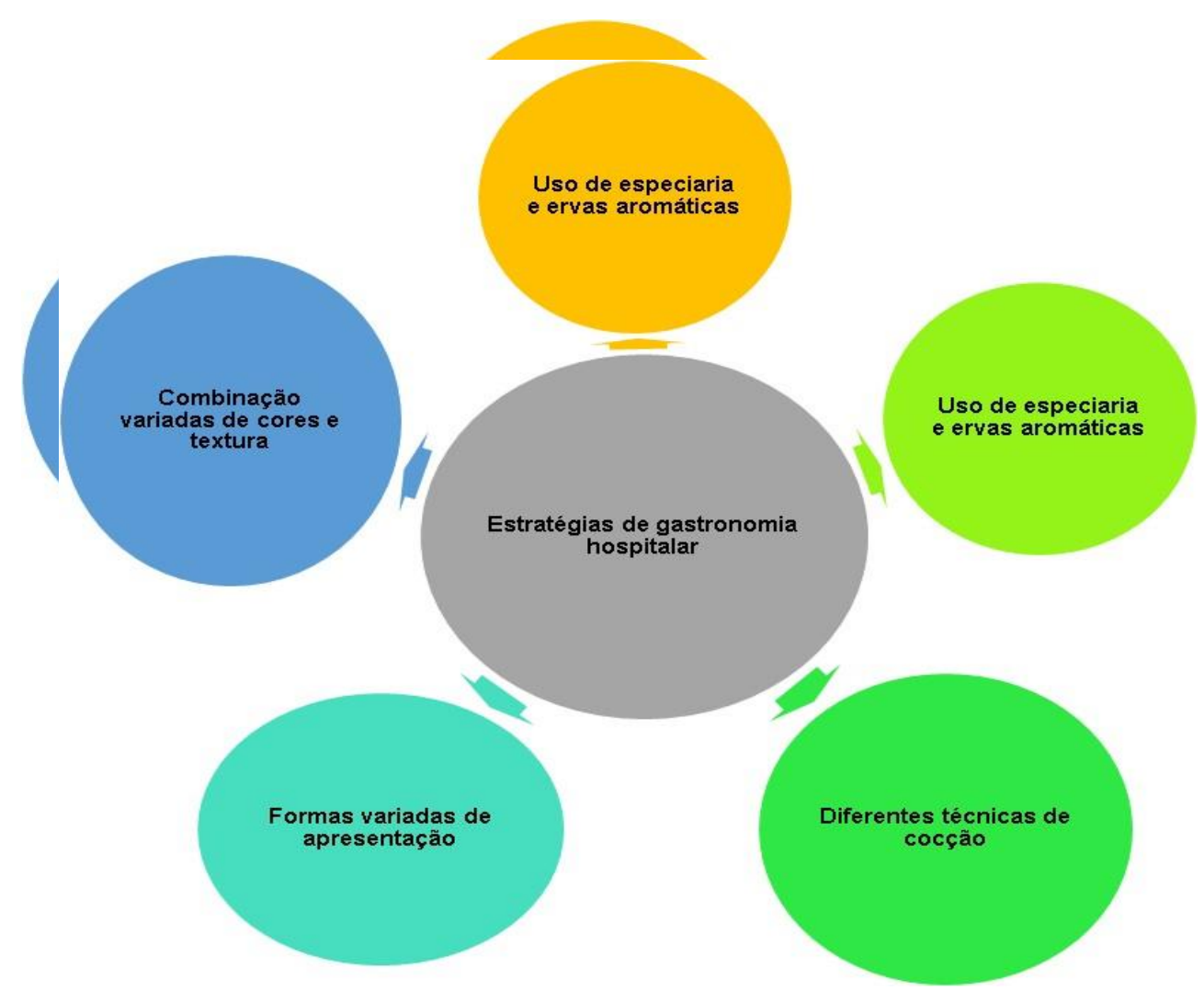




\subsection{DESNUTRIÇÃO HOSPITALAR}

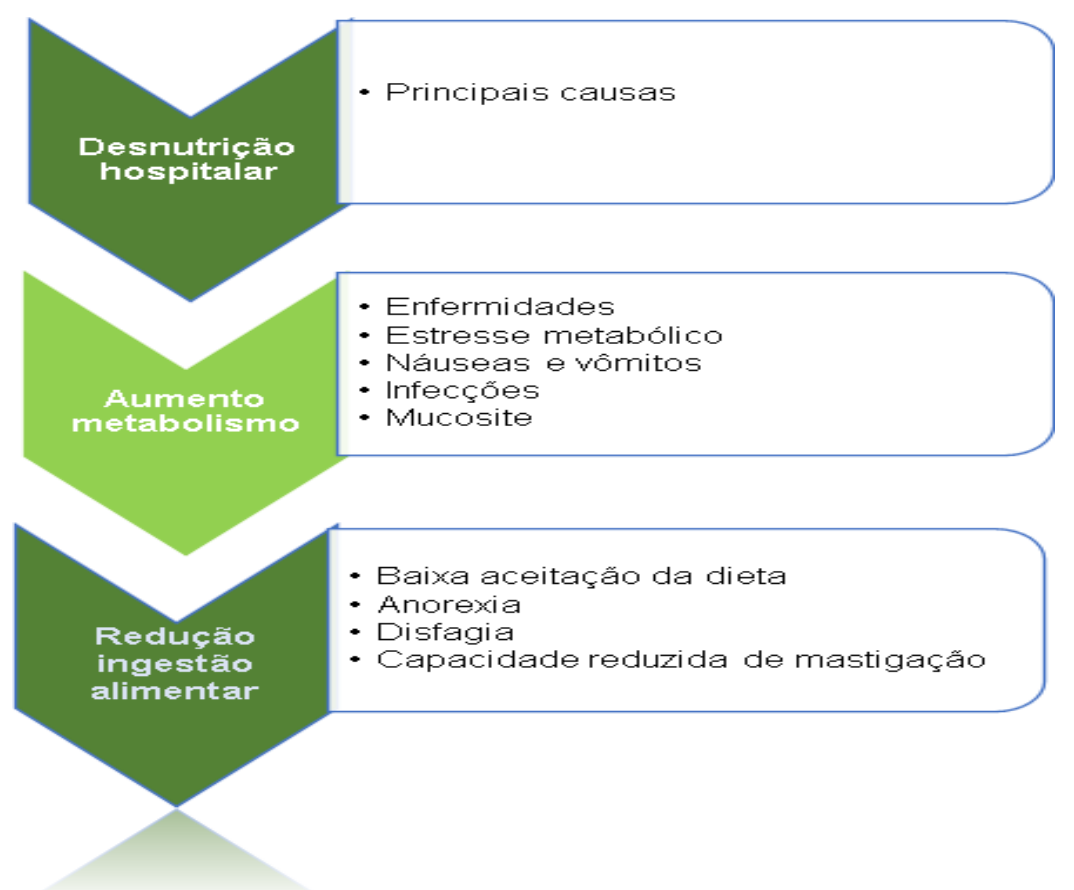

A desnutrição hospitalar gera um agravo multifatorial a qual atinge o estado nutricional e de saúde do paciente que se encontra com alguma patologia clínica (Lew et al., 2017).

A internação é um período em que o indivíduo está afastado de suas atividades e funções realizadas no seu meio social, com isso pode apresentar alterações psicológicas, falta de apetite, recusa e desinteresse alimentar, consequentemente, eles podem sofrer perda de peso, desnutrição, caquexia e resultados da baixa ingestão dietética. Portanto a terapia nutricional em ambiente hospitalar tem como principal objetivo garantir a quantidade e qualidade de nutrientes necessários a cada indivíduo, além de auxiliar no tratamento para recuperação da saúde dos pacientes internados e é responsável também por amenizar o sofrimento gerado no período de internação (NASCIMENTO; et $\mathrm{al}, 2017)$.

A impossibilidade de fornecer nutrientes necessários para atender às exigências corporais é uma preocupação séria em pacientes hospitalizados, principalmente pessoas com doenças crônicas, ferimentos traumáticos, idosos epacientes internados em Unidade de Terapia 
Intensiva (UTI) que são especialmente vulneráveis às complicações decorrentes da desnutrição, pois eles podem apresentar limitação da ingesta hídrica, instabilidade hemodinâmica e diminuição da absorção de drogas e nutrientes. Além desses fatores, a pouca atenção dos profissionais de saúde na avaliação nutricional e a monitoração ineficaz da aceitação da dieta podem contribuir para alterações noestado nutricional. Todos esses fatores se refletem na ausência de rastreamento e triagem nutricional dos pacientes em ambiente hospitalar (BERHMAM; LIMA, 2019).

\section{CONCLUSÃO}

A alimentação acompanha o desenvolvimento do ser humano durante toda sua vida, é o elo entre o indivíduo e o ambiente em que se encontra. A ocorrência dadoença e a necessidade de internação priva o paciente de sua relação alimentar habitual. A gastronomia hospitalar hoje é uma ferramenta de bem estar e de promoção de qualidade de vida e fundamental ao avanço da qualidade no atendimento hospitalar. Conclui-se que a gastronomia hospitalar pode ser utilizada como uma aliada na recuperação do estado nutricional de pacientes hospitalizados desnutridos, melhorando as características sensoriais das preparações, a apresentação das dietas e o atendimento aos pacientes.

Entretanto, são necessários mais estudos de campo que comprovem esse benefício. Dessa forma, além de tornar o hospital um ambiente mais acolhedor e minimizar o impacto da internação sobre os pacientes, esses novos conceitos poderão ser aplicados de forma a contribuir para a redução da desnutrição hospitalar. Estudos dessa natureza podem apontar novas abordagens na dimensão do cuidado integral, em alimentação, nutrição e saúde hospitalar. Podem, ainda, contribuir com os profissionais envolvidos com a alimentação, para que suas ações considerem a subjetividade dos pacientes. Tendo em vista todos os aspectos referidos, aliado à pouca produção de trabalhos com essa temática, destaca-se a relevância dessa proposta para diferentes campos do conhecimento, com ênfase no setor de alimentação coletiva hospitalar. 
O ser humano sempre procura se alimentar de acordo com as suas preferências, e uma boa qualidade sensorial é fator primordial para o consumo deum alimento, visto que o indivíduo não se alimenta pensando apenas nos benefícios nutricionais que terá a partir daquela refeição.

Para o paciente hospitalizado, a alimentação é essencial para suprir as necessidades nutricionais e auxiliar na recuperação/manutenção do estado nutricional. Diante do apresentado, conclui-se, portanto, a validação da hipótese visto que, uma dieta com boa qualidade sensorial influencia de maneira positiva na aceitação por parte do paciente hospitalizado, da dieta oferecida. Conseguinte, há contribuição para melhora do estado nutricional, imunidade, diminuição da dor e angústia oriundas da doença e do período de internação. Sendo assim, a gastronomia deve ser um dos aspectos principais das dietas hospitalares, visando aliar as necessidade e restrições alimentares do paciente a uma refeição atraente e agradável aos olhos e ao paladar.

A aceitação e satisfação por parte do paciente da dieta hospitalar estão intimamente relacionadas à qualidade sensorial da refeição oferecida. As dietas hospitalares devem considerar a relação do paciente com o alimento, tendo como

prioridade suas preferências e hábitos individuais, portanto a escolha dos alimentos que estimulam e satisfazem o paladar é uma escolha prática. (LAGES, 2017).

\section{REFERÊNCIAS}

BEHRMANN, G.; LIMA, A. M. P. (2019). Relevância do Protocolo em Nutrição na Avaliação do Estado Nutricional do Paciente Hospitalizado: Uma Revisão Integrativa. Revista Da Associação Brasileira De Nutrição - ASBRAN, ıo(I), 134-I4I. Recuperado de https://www.rasbran.com.br/rasbran/article/view/ıIo3

CAMPOS JUNIOR, C.G. et al. Aceitabilidade da dieta hospitalar de um hospital municipal da cidade de Cuiabá, Mato Grosso. 2019. I2 f. TCC (Graduação em Nutrição) Centro Universitário de Várzea Grande (UNIVAG (2019)

DELGADO, T. C. G. A importância da nutrição no cuidado ao paciente desnutrido. In: ENCONTRO NACIONAL DE PÓS GRADUAÇÃO, I, 2017, Santos. Anais Eletrônicos. Santos: UNISANTA, 2017 .

DEMÁRIO R. L., SOUSA A. A., SALLES R.K. Comida de hospital: percepções de pacientes em um hospital público com uma proposta de atendimento humanizado. Cien Saude Colet. 2017 Jan/Jun;15(I):1275-82 
LAGES, P. C.; RIBEIRO, R. C.; SOARES, L. S.. A gastronomia como proposta de qualidade dietética das refeições hospitalares pastosas: Análise, intervenção e avaliação. Rev. Alimentos e Nutrição / Brazilian Journal of Food and Nutrition, Araraquara, v. 24, n. I, p. 93-99, jan. /mar., 2017 .

LEW, C. C. H.; YANDELL, R.; FRASER, R. J. L.; CHUA, A. P.; CHONG, M. F. F.; MILLER, M. (2017). Association Between Malnutrition and Clinical Outcomes in the

Intensive Care Unit: A Systematic Review. JPEN J Parenter Enteral Nutrition 4I(5), 744758. https://doi:10.1177/or48607115625638

MARTINS, P.; BARATTO, I. Gastronomia Hospitalar: Treinamento em bases de cozinha. RBONE - Revista brasileira de obesidade, nutrição e emagrecimento, v. I2, n. 69, p. IIo - II7, 2018.

MEDILINE, essenciais Literature Analysis And Retrieval System online/PubMed.

MENDES, M. S. A; MACHADO, C. C. B; SOUZA, A. V. Pesquisa de satisfação com dietas hospitalares servidas no almoço da clínica médica de um hospital público de Goiânia, GO. Revista DEMETRA: Alimentação, Nutrição \& Saúde, Rio de Janeiro, v. I4, p. I-II, 2019.

PAIVA, A. C. M. S. Gastronomia e nutrição: perspectiva pessoal e profissional. Revista Iberoamericana de Estudos em Educação, v. 13, n. 2, p. 748 - 763, abr./jun. 2018.

PINTO, C. C.; ALVES, E. A. A gastronomia no contexto da hotelaria hospitalar: um estudo de caso na cidade do rio de janeiro, RJ, BRASIL. RAHIS, Belo Horizonte, v. I4, n. 2, p. I - 13, 2017.

NASCIMENTO, T. et al. Aceitabilidade das dietas orais de hospital do Sul de Minas. Revista UIIPS, São Paulo, v.5, n.5, p. I4I-I48, 2017.

SANTOS, V. S.; GONTIJO, M. C. F.; ALMEIDA, M. E. F. Efeito da gastronomia na aceitabilidade de dietas hospitalares. Nutrición clínica e dietética hospitalaria, v. 37, n. 3, p. $17-22,2017$.

SILVA, A. S. Aceitabilidade da dieta para pacientes hipertensos e diabéticos internados em um hospital público no recôncavo da Bahia. 2018.

SOUZA BS, MOLERO MP, MOLINA VBC. Gastronomia e humanização hospitalar. Revista Multidisciplinar da Saúde (RMS), v. 3, n.oI, ano 2021, p. I4-26.

SOUZA, Mariana Delega de; NAKASATO, Miyoko. A gastronomia hospitalar auxiliando na redução dos índices de desnutrição de pacientes hospitalizados. Rev. O Mundo da Saúde, São Paulo, v. 35, n. 2, p. 208-214, 2017 .

SPENCE, C. Hospital food. Flavour, v.6, n.3, 2017 . 